BMJ Open

Diabetes

Research

\& Care

\title{
Clinical characteristics, complications, comorbidities and treatment patterns among patients with type 2 diabetes mellitus in a large integrated health system
}

Kevin M Pantalone, ${ }^{1}$ Todd M Hobbs, ${ }^{2}$ Brian J Wells, ${ }^{3}$ Sheldon X Kong, ${ }^{4}$
Michael W Kattan, ${ }^{5}$ Jonathan Bouchard, ${ }^{4}$ Changhong Yu, ${ }^{5}$ Brian Sakurada,
Alex Milinovich, ${ }^{5}$ Wayne Weng, ${ }^{4}$ Janine M Bauman, ${ }^{5}$ Robert S Zimmerman ${ }^{1}$

\section{ABSTRACT}

Purpose: To compare the prevalence of diabetesrelated complications and comorbidities, clinical characteristics, glycemic control, and treatment patterns in patients with type 2 diabetes (T2D) within a large integrated healthcare system in 2008 vs 2013. Methods: An electronic health record system was used to create a cross-sectional summary of all patients with T2D as on 1 July 2008 and 1 July 2013. Differences between the two data sets were assessed after adjusting for age, gender, race, and household income.

Results: In 2008 and 2013, 24493 and 41582

Received 18 February 2015 Revised 11 June 2015 Accepted 26 June 2015

\section{CrossMark}

For numbered affiliations see end of article.

Correspondence to Dr Kevin M Pantalone; pantalk@ccf.org patients with T2D were identified, respectively, of which the majority were male $(52.3 \%$ and $50.1 \%)$ and Caucasian (79\% and $75.2 \%)$. The mean ages (years) were 64.8 and 64.3 . The percentages of patients across the defined A1C categories were 64.3 and 66.7 for $<7 \%, 21.1$ and 18.8 for $7-7.9 \%, 7.8$ and 7.5 for $8-8.9 \%$, and 6.8 and 7.0 for $\geq 9 \%$ in 2008 and 2013 , respectively. The most prevalent T2D-related comorbidities were hypertension $(82.5 \%$ and $87.2 \%)$ and cardiovascular disease $(26.9 \%$ and $22.3 \%)$ in 2008 and 2013, respectively. Thiazolidinedione and sulfonylurea use decreased, whereas metformin and dipeptidyl peptidase-4 inhibitor use increased in the 5-year period.

Conclusions: Patients with T2D are characterized by a high number of comorbidities. Over $85 \%$ of the patients had an $\mathrm{A} 1 \mathrm{C}<8 \%$ within our integrated health delivery system in 2008 and 2013. In 2008 and 2013, metformin therapy was the most commonly utilized antidiabetic agent, and sulfonylureas were the most commonly utilized oral antidiabetic agent in combination with metformin. As integrated health systems assume greater shared financial risk in newer payment models, achieving glycemic targets (A1C) and the management of comorbidities will become evermore important, for preventing diabetes-related complications, as well as to ensure reimbursement for the medical care that is rendered to patients with diabetes.

\section{Key messages}

- While the prevalence of hypertension has increased among patients with type 2 diabetes, the prevalence of other comorbidities decreased in 2013 when compared with 2008.

- The treatment patterns and choice of antidiabetic therapies were observed to change in 2013 vs 2008. Thiazolidinedione and sulfonylurea use has decreased, whereas metformin and dipeptidyl peptidase-4 inhibitor use increased in the 5-year period.

- Over $85 \%$ of the patients had an $\mathrm{A} 1 \mathrm{C}<8 \%$ within our integrated health delivery system in 2008 and 2013.

\section{Research questions}

What are the key barriers that prevent obtaining glycemic control in patients who continue to have a persistently elevated $\mathrm{A} 1 \mathrm{C}$ ?

- How can electronic health record systems be leveraged to further improve diabetes-related outcomes?

- How can we further improve the quality of care we render to patients with diabetes?

- How have the clinical characteristics, complications, comorbidities, and treatment patterns among patients with new-onset type 2 diabetes mellitus, managed within in a large integrated health system, changed over time?

\section{INTRODUCTION}

The prevalence of diagnosed diabetes has more than doubled over the past two decades, from $3.8 \%$ in 1990 to $8.6 \%$ in 2010. ${ }^{1}$ Research using the USA National Health and Nutrition Examination Survey (NHANES) database reported that from 
2003 to 2006 only $57 \%$ of people with diabetes, largely patients with type 2 diabetes (T2D), had a glycosylated hemoglobin (HbA1C) <7\%. ${ }^{2}{ }^{3}$ Recently, from 2007 to 2010, the percentage of patients under adequate control was noted to be even lower, with only $52.5 \%$ of people with diabetes achieving an $\mathrm{A} 1 \mathrm{C}<7.0 \%{ }^{4}$ This is a matter of concern, as inadequate glycemic control not only plays a role in cardiovascular risk, but it also remains the leading cause of blindness, kidney failure, and nontraumatic lower limb amputations in the USA. ${ }^{5}$ While reports have suggested that we continue to make progress in attaining glycemic targets and managing comorbidities, in addition to reducing the rates of diabetes-related complications, there is still substantial room for improvement. ${ }^{6} 7$

Over the past few decades, integrated health delivery systems have evolved, and the way that medical care is delivered has changed considerably. Integrated health delivery systems are organized, coordinated, and collaborative networks which link various healthcare providers to provide a coordinated, vertical continuum of services to a particular patient population or community. ${ }^{8}$ Currently, there are limited real-world data describing the management of the patient population with T2D within an integrated health system. Given the variety of therapies available to treat patients with T2D, it is important to evaluate the current patterns of management and the associated clinical outcomes. The objective of this cross-sectional analysis was to analyze and compare the prevalence of diabetes-related complications and comorbidities, as well as the clinical characteristics, glycemic control, and treatment patterns in patients with T2D within a large integrated health system in 2008 vs 2013.

\section{RESEARCH DESIGN AND METHODS}

The electronic health record (EHR) system at Cleveland Clinic was used to create a cross-sectional summary of patients with T2D at two different time points (1 July 2008 and 1 July 2013). Cleveland Clinic utilizes EPIC My Practice across the entire organization. Patients with T2D were identified according to the EMERGE algorithm published by Kho et al. ${ }^{9}$ All patients with documented International Classification of Diseases, Ninth Revision (ICD-9) codes for T1D were excluded. We included all patients with ICD-9 codes of $250 . x 0$ or 250 . x2, except for codes 250.10 and 250.12, as these are indicative of T2D with ketoacidosis, a condition that closely resembles that of T1D. Additional patients with T2D were identified via the algorithm if they had been prescribed a non-insulin T2D therapy, had at least two encounters with a T2D diagnosis entered into the EHR by a clinician, or had an abnormal glucose level consistent with a diagnosis of diabetes (but no documented ICD-9 code for T1D) as per the most recent American Diabetes Association (ADA) criteria (fasting blood glucose $(\mathrm{BG}) \geq 126 \mathrm{mg} / \mathrm{dL}, \mathrm{HbA} 1 \mathrm{C} \geq 6.5 \%$, or random $\mathrm{BG} \geq 200 \mathrm{mg} / \mathrm{dL})^{10}$
Both inpatient and outpatient HbA1C values (laboratory and point of care) were included, but for fasting and random glucose values, only outpatient laboratory values were utilized. If the BG values were not ordered specifically as fasting, they were assumed fasting if obtained simultaneously with a lipid panel. All BG values obtained at the point of care, or as part of a glucose tolerance test, were excluded.

The following T2D medication classes were utilized as a means of identifying patients with T2D: biguanide (metformin), sulfonylureas, thiazolidinediones, $\alpha$-glucosidase inhibitors, meglitinides, dipeptidyl peptidase-4 (DPP-4) inhibitors, glucagon-like peptide-1 (GLP-1) agonists and insulin. The remaining classes of antidiabetic medications were excluded either because they were too new (sodium glucose transporter-2 inhibitors) or non-specific for diabetes (colesevelam), or there were too few patients receiving the medication (pramlintide, bromocriptine (Cycloset)). Patient characteristics, complications, and comorbidities were extracted from the EHR dating back to 1998. Active medications were based on the current medication list as on 1 July 2008 and 1 July 2013. Laboratory values within 1 year prior to the index dates were included in the data sets. For multiple eligible values for the same patient, the most recent value prior to the index date was used. The Diabetes Complication Severity Index ${ }^{11}$ was calculated and reported for the overall 2008 and 2013 T2D populations.

The analyses compared characteristics of patients in 2008 vs 2013 while adjusting for age, gender, race, and income (median household income by census block group derived from the 2010 US census). These four variables were chosen because they are all unmodifiable from the perspective of the clinician. The years of study, 2008 and 2013, were chosen because it was 2008 when medication reconciliation was mandated to occur at the transitions of care at our institution, and 2013 was the point in time at which the data were extracted from the EHR for analysis. The adjustment was performed using generalized estimating equations (GEE) to account for the clustering caused by the inclusion of some patients in the 2008 and 2013 data sets. Separate GEE models were fit for each of the comparison variables as outcomes using age, gender, race, income, and year as covariates. The models were used to obtain the adjusted values reported in tables 1-3.

The adjusted analysis compares the theoretical characteristics of patients in 2008 vs 2013 if the two populations were composed of similar patients in terms of age, gender, race, and income. Statistical significance between 2008 and 2013 was calculated by fitting individual regression models for each of the variables of interest as the dependent variable and age, gender, race, income, and year as the independent variables. In these models, a statistically significant coefficient for year indicates a difference in values between 2008 and 2013 after adjustment. The independent variables (age, gender, race, and income) were used to construct the regression models for comparing 2008 vs 2013. Univariate, non- 
Table 1 Clinical characteristics, comorbidities, complications, and diabetes-related health maintenance at index (N, \%)

\begin{tabular}{|c|c|c|c|c|c|}
\hline \multirow[b]{2}{*}{ Variable } & \multicolumn{2}{|c|}{$\begin{array}{l}2008 \\
\text { Index year } \\
\mathrm{N}=24493\end{array}$} & \multicolumn{2}{|c|}{$\begin{array}{l}2013 \\
\text { Index year } \\
\mathrm{N}=41582\end{array}$} & \multirow[b]{2}{*}{ p Value } \\
\hline & $\mathbf{N}$ & Per cent & $\mathbf{N}$ & Per cent & \\
\hline Male & 12802 & 52.3 & 20852 & 50.1 & $<0.0001^{*}$ \\
\hline \multicolumn{6}{|l|}{ Race } \\
\hline Caucasian & 18907 & 79 & 31251 & 75.2 & $<0.0001^{*}$ \\
\hline Black & 3420 & 14.3 & 8189 & 19.7 & \\
\hline Other & 1616 & 6.7 & 2102 & 5.1 & \\
\hline Missing & 550 & & 40 & & \\
\hline Smoking & 8806 & 55.0 & 21108 & 53.3 & $0.0002 \dagger$ \\
\hline Flu shot last flu season & 8008 & 32.7 & 16137 & 38.8 & $<0.0001 \dagger$ \\
\hline Pneumonia vaccine in past 10 years & 12969 & 51.8 & 25673 & 61.7 & $<0.0001 \dagger$ \\
\hline \multicolumn{6}{|l|}{ A1c } \\
\hline$<7 \%$ & 13223 & 64.3 & 23885 & 66.7 & $<0.0001 \dagger$ \\
\hline $7-7.9 \%$ & 4326 & 21.1 & 6726 & 18.8 & \\
\hline $8-8.9 \%$ & 1597 & 7.8 & 2679 & 7.5 & \\
\hline$>9 \%$ & 1403 & 6.8 & 2521 & 7.0 & \\
\hline Statin & 16427 & 67.1 & 28503 & 68.5 & $<0.0001 \dagger$ \\
\hline ASA & 12556 & 51.3 & 22285 & 53.6 & $<0.0001 \dagger$ \\
\hline ARB or ACE & 17001 & 69.4 & 28741 & 69.1 & $0.0001 \dagger$ \\
\hline GFR<60 mL/min $\ddagger$ & 3978 & 18.8 & 5977 & 16.3 & $<0.0001 \dagger$ \\
\hline Hypertension & 20208 & 82.5 & 36250 & 87.2 & $<0.0001 \dagger$ \\
\hline Retinopathy & 790 & 3.2 & 1425 & 3.4 & $0.1146 \dagger$ \\
\hline Nephropathy & 6565 & 26.8 & 10753 & 25.9 & $0.56 \dagger$ \\
\hline Neuropathy & 4770 & 19.5 & 7493 & 18.0 & $<0.0001 \dagger$ \\
\hline Cerebrovascular disease & 2374 & 9.7 & 3348 & 8.1 & $<0.0001 \dagger$ \\
\hline Cardiovascular disease & 6581 & 26.9 & 9273 & 22.3 & $<0.0001 \dagger$ \\
\hline Peripheral vascular disease & 1315 & 5.4 & 2049 & 4.9 & $<0.0001 \dagger$ \\
\hline
\end{tabular}

${ }^{*}$ Compared using Pearson's $\chi^{2}$ test.

†Adjusted for sex, age, race, and income using generalized estimating equations.

‡GFR calculated via CKD-EPI.

ARB, angiotensin receptor blocker; ASA, acetylsalicylic acid; CKD-EPI, Chronic Kidney Disease Epidemiology Collaboration; GFR, glomerular filtration rate.

parametric tests were used to compare age, gender, race, and income.

For the purpose of this analysis, in order to exclude patients whose T2D was not actively being managed by providers within the Cleveland Clinic Health System, patients were required to have had at least one visit with an endocrinologist or primary care provider (family medicine, internal medicine) with an encounter diagnosis of diabetes mellitus (DM) (250.XX) at least 1 year prior to the index dates (ie, 1 July 2008 and 1 July 2013, respectively).

Table 2 Clinical characteristics, comorbidities, complications, and diabetes-related health maintenance at index (mean, SD)

\begin{tabular}{|c|c|c|c|c|c|}
\hline \multirow[b]{2}{*}{ Variable } & \multicolumn{2}{|c|}{$\begin{array}{l}2008 \\
\text { Index year } \\
\mathrm{N}=24493\end{array}$} & \multicolumn{2}{|c|}{$\begin{array}{l}2013 \\
\text { Index year } \\
\mathrm{N}=41582\end{array}$} & \multirow[b]{2}{*}{ p Value } \\
\hline & Mean & SD & Mean & SD & \\
\hline Age at index dates (years) & 64.8 & 12.9 & 64.3 & 12.9 & $<0.0001^{*}$ \\
\hline $\mathrm{BMI}\left(\mathrm{kg} / \mathrm{m}^{2}\right)$ & 32.5 & 7.0 & 32.8 & 7.0 & $<0.0001 \dagger$ \\
\hline Systolic blood pressure (mm Hg) & 129.5 & 17.4 & 129.8 & 17.4 & $0.063 \dagger$ \\
\hline Diastolic blood pressure $(\mathrm{mm} \mathrm{Hg})$ & 74.2 & 10.2 & 74.5 & 10.3 & $0.007 \dagger$ \\
\hline LDL cholesterol (mg/dL) & 92.1 & 32.6 & 88.0 & 32.7 & $<0.0001 \dagger$ \\
\hline DCSI & 1.5 & 1.3 & 1.4 & 1.6 & $<0.0001 \dagger$ \\
\hline Number of diabetes visits in prior year $(\mathrm{N}) \ddagger$ & 2.6 & 1.6 & 2.5 & 1.6 & $<0.0001 \dagger$ \\
\hline Number of canceled visits in prior year $(\mathrm{N})$ & 1.2 & 1.9 & 1.5 & 1.9 & $<0.0001 \dagger$ \\
\hline Median household income (US\$1000) & 59.4 & 23.5 & 56.9 & 23.4 & $<0.0001^{*}$ \\
\hline
\end{tabular}

${ }^{*}$ Compared using the Wilcoxon Mann-Whitney test.

†Adjusted for sex, age, race, and income using generalized estimating equations.

$\ddagger$ Endocrinology or primary care visits.

BMI, body mass index; DCSI, Diabetes Complications Severity Index; LDL, low-density lipoprotein. 


\begin{tabular}{|c|c|c|c|c|c|}
\hline & \multicolumn{2}{|c|}{$\begin{array}{l}2008 \\
\text { Index year* } \\
\mathrm{N}=24493\end{array}$} & \multicolumn{2}{|c|}{$\begin{array}{l}2013 \\
\text { Index year* } \\
\mathrm{N}=41582\end{array}$} & \multirow{2}{*}{$\begin{array}{l}\text { Adjusted } \\
\text { p value }\end{array}$} \\
\hline & $\mathbf{N}$ & Per cent & $\mathbf{N}$ & Per cent & \\
\hline$\alpha$-Glucosidase inhibitors & 101 & 0.4 & 130 & 0.3 & 0.1190 \\
\hline Biguanides (metformin) & 15425 & 63.0 & 27987 & 67.3 & $<0.0001$ \\
\hline Metformin monotherapy & 5640 & 23.0 & 11735 & 28.2 & $<0.0001$ \\
\hline Metformin+sulfonylurea† & 3313 & 13.5 & 5565 & 13.3 & 0.62 \\
\hline Metformin+thiazolidinedione & 963 & 3.9 & 422 & 1.0 & $<0.0001$ \\
\hline Metformin+dipeptidyl peptidase-4 inhibitor† & 402 & 1.6 & 1580 & 3.8 & $<0.0001$ \\
\hline Metformin+GLP-1† & 153 & 0.6 & 264 & 0.6 & 0.86 \\
\hline Metformin+insulin† & 411 & 1.7 & 1178 & 2.8 & $<0.0001$ \\
\hline Dipeptidyl peptidase-4 inhibitors & 1952 & 8.0 & 6566 & 15.8 & $<0.0001$ \\
\hline Glucagon-like peptide-1 agonists & 984 & 4.0 & 1563 & 3.8 & $<0.0001$ \\
\hline Meglitinides & 247 & 1.0 & 212 & 0.5 & $<0.0001$ \\
\hline Sulfonylureas & 9898 & 40.4 & 14705 & 35.4 & $<0.0001$ \\
\hline Thiazolidinediones & 4849 & 19.8 & 2852 & 6.9 & $<0.0001$ \\
\hline Antidiabetic combination therapy $\ddagger$ & 11286 & 46.0 & 18232 & 43.8 & $<0.0001$ \\
\hline Statin & 16427 & 67.1 & 28503 & 68.5 & $<0.0001$ \\
\hline Aspirin & 12556 & 51.3 & 22285 & 53.6 & $<0.0001$ \\
\hline ACE or ARB & 17001 & 69.4 & 28741 & 69.1 & 0.0001 \\
\hline Insulin total & 3711 & 15.2 & 7832 & 18.8 & $<0.0001$ \\
\hline Human & 1035 & 27.9 & 1353 & 17.3 & $<0.0001$ \\
\hline Analog (basal or bolus) & 3070 & 82.7 & 7066 & 90.2 & $<0.0001$ \\
\hline Basal & 3474 & 93.6 & 7522 & 96.0 & $<0.0001$ \\
\hline Bolus & 2205 & 59.4 & 4583 & 58.5 & 0.0003 \\
\hline Premix & 515 & 13.9 & 150 & 1.9 & $<0.0001$ \\
\hline $\begin{array}{l}\text { Insulin+any number of oral antidiabetic medication } \\
\text { classes§ }\end{array}$ & 2194 & 9.0 & 4544 & 10.9 & $<0.0001$ \\
\hline Insulin+one oral antidiabetic medication class & 854 & 3.5 & 2032 & 4.9 & $<0.0001$ \\
\hline
\end{tabular}

This study was approved by the Cleveland Clinic's Institutional Review Board.

\section{RESULTS}

The 2008 and 2013 data sets included 24493 and 41582 patients with T2D, respectively. Of note, 12728 (52.0\%) patients included in the 2008 data set were also included in the 2013 data set.

\section{Characteristics (categorical and continuous variables)}

The majority of patients with T2D were male and Caucasian $(52.3 \%$ and $50.1 \%, \mathrm{p}<0.0001)$ and $(79 \%$ and $75.2 \%, \mathrm{p}<0.0001)$, in the 2008 and 2013 data sets, respectively. The mean age (years) was $64.8 \pm 12.9$ in 2008 , and $64.3 \pm 12.9$ in 2013 , $\mathrm{p}<0.0001$. The mean household income (in US\$1000) was observed to be higher in 2008 vs $2013,59.4$ vs $56.9, \mathrm{p}<0.0001$. The percentage of active smokers was found to be $55 \%$ in 2008 , and $53.3 \%$ in $2013(\mathrm{p}=0.0002)$ (table 1). Small, non-clinically significant changes were observed between 2008 and 2013, respectively, for mean body mass index (32.5 and 32.8; $\mathrm{p}<0.0001)$, systolic blood pressure (BP) (129.5 and 129.8; $\mathrm{p}=0.063)$, diastolic BP (74.2 and 74.5; $\mathrm{p}=0.007)$, and low-density lipoprotein (LDL) cholesterol (92.1 and 88; $\mathrm{p}<0.0001$ ) (table 2).

\section{Comorbidities}

The percentages (\%) of patients with T2D with comorbidities in 2008 and 2013, respectively, were glomerular filtration rate $<60 \mathrm{~mL} / \mathrm{min}$, calculated by the CKD-EPI (Chronic Kidney Disease Epidemiology Collaboration) formula (18.8 and $16.3 ; \mathrm{p}<0.0001)$, hypertension (HTN) (82.5 and 87.2; $<<0.0001)$, cerebrovascular disease (9.7 and 8.1; $\mathrm{p}<0.0001)$, cardiovascular disease (CVD) (26.9 and 22.3; $\mathrm{p}<0.0001$ ), and peripheral vascular disease (5.4 and 4.9; $\mathrm{p}<0.0001$ ) (table 1 ). The Diabetes Complications Severity Index (DCSI) scores $($ mean $\pm \mathrm{SD}$ ) for patients with T2D in 2008 and 2013 were 1.5 and $1.4 ; \mathrm{p}<0.0001$, respectively (table 2 ). 


\section{Microvascular complications}

The percentages $(\%)$ of patients with T2D with microvascular complications in 2008 and 2013, respectively, were retinopathy (3.2 and $3.4 ; \mathrm{p}=0.11$ ), nephropathy (26.8 and 25.9; $\mathrm{p}=0.56$ ), and neuropathy (19.5 and 18.0; $\mathrm{p}<0.0001)$ (table 1$)$.

\section{Diabetes-related follow-up and health maintenance}

In 2008 and 2013, the mean numbers of diabetes-related office visits were 2.6 and 2.5; $<<0.0001$, and of missed appointments were $1.2 \pm 1.9$ and $1.5 \pm 1.9 ; \mathrm{p}<0.0001$, respectively (table 2 ). The percentages $(\%)$ of patients, in 2008 and 2013, who received the recommended influenza vaccine in the prior season and pneumonia vaccine within the past 10 years, were 32.7 and 38.8 and 51.8 and 61.7, respectively (table 1 ). Dilated eye examinations were not tracked by the EHR in 2008. This has since become more standard practice, yet the number of patients with the structured documentation of yearly dilated eye examinations remains low; only $12.9 \%$ of patients with diabetes in 2013 had a dilated eye examination documented in the structured health maintenance section of the EHR.

\section{Glycemic control}

The population was divided into four groups based on the index A1C level. The percentages of patients across the defined A1C categories were 64.3 and 66.7 for $<7 \%$, 21.1 and 18.8 for $7-7.9 \%, 7.8$ and 7.5 for $8-8.9 \%$, and 6.8 and 7.0 for $\geq 9 \%$ in 2008 and 2013, respectively $(\mathrm{p}<0.0001)$, (table 1$)$.

\section{Medications}

In the 2008 and 2013 data sets, the most commonly utilized antidiabetic agents were metformin and sulfonylureas. The percentages of patients receiving metformin overall and metformin monotherapy in 2008 and 2013, were 63.0 and 67.3, $\mathrm{p}<0.0001$ and 23.0 and 28.2, $\mathrm{p}<0.0001$, respectively. Sulfonylureas were the most commonly utilized oral antidiabetic agent as two-drug combination therapy with metformin in 2008 and 2013 (13.5 and 13.3, $\mathrm{p}=0.62$ ). The percentages of patients in 2008 and 2013 receiving two-drug combination therapy with metformin and a thiazolidinedione, a DPP-4 inhibitor, a GLP-1 agonist, or insulin were 3.9 and 1.0, $\mathrm{p}<0.0001,1.6$ and $3.8, \mathrm{p}<0.0001,0.6$ and $0.6, \mathrm{p}=0.62$, and 1.7 and 2.8, $\mathrm{p}<0.0001$ (table 3 ).

The most significant differences from 2008 to 2013 in the per cent of patients using antidiabetic medications were observed for those who were prescribed thiazolidinediones $(19.8 \%$ to $6.9 \%, \mathrm{p}<0.0001)$, and DPP-4 inhibitors $(8.0 \%$ and $15.8 \% ; \mathrm{p}<0.0001)$. Sulfonylurea usage (overall) fell from $40.4 \%$ to $35.4 \%$; $\mathrm{p}<0.0001$. Insulin usage occurred in only $15.2 \%$ and $18.8 \%$ of patients, $\mathrm{p}<0.0001$, while combination antidiabetic therapy (any 2 of the 8 medication classes) was used by $46.0 \%$ and $43.8 \%$ of patients, $\mathrm{p}<0.0001$, in the respective years. Utilization of GLP-1 agonist therapy remained low at both index dates $(\leq 4 \%)$. The distribution of the remaining prescribed antidiabetic medications (\% of patients receiving the therapy) is shown in table 3 .

Regarding the patients receiving insulin therapy, the percentage $(\%)$ of patients receiving human insulin decreased and that of analog insulin usage correspondingly increased from 2008 to 2013. Usage of basal or bolus insulin changed only slightly from 2008 to 2013, whereas the use of premixed insulin was observed to decrease rather significantly from 2008 vs 2013 (13.9 vs $1.9 ; \mathrm{p}<0.0001)$. The percentages of patients receiving insulin in combination with any one oral antidiabetic class, or any number of oral antidiabetic classes, were 3.5 and 4.9, $\mathrm{p}<0.0001$ and 9.0 and 10.9, p<0.0001, in 2008 and 2013, respectively.

The majority of patients with T2D were receiving a statin, aspirin (acetylsalicylic acid), and an ACE inhibitor or angiotensin receptor blocker, with small, albeit significant changes from 2008 to 2013.

A random chart review conducted on 100 participants supported the validity of the extraction of structured data (ICD-9 codes, medications, laboratory values, etc.).

Please see tables 1-3 for a comprehensive summary of the data provided.

\section{DISCUSSION}

Over the past few decades, integrated health delivery systems have evolved, and continue to increase in number. As a result of the evolution of these systems, the way that diabetes-related care is delivered and management of its comorbid conditions have changed considerably. This report describes the population of patients with T2D receiving care within the integrated healthy delivery system at Cleveland Clinic, which uses a single EHR system across the entire organization (EPIC). The EHR data extraction and analysis were conducted to better identify the population of patients with T2D who are receiving care in an integrated health system. Currently, the literature only includes large studies using the general population of patients with T2D, as characterized regularly via NHANES, a stratified, multistage, probability cluster survey conducted in the non-institutionalized US population.

We were able to further characterize how the profile of patients with T2D within the EHR has changed with time (ie, 2008 vs 2013). There was an increase in the number of participants who were female, Black, and from a lower socioeconomic status (SES), as per the median household income data derived from the last US census. Patients with diabetes were found to be younger in 2013 vs 2008 from a statistical standpoint; however, the very small difference in age noted is of no clinical relevance.

In terms of glycemic control, there was a higher percentage of patients with an $\mathrm{A} 1 \mathrm{C}<7 \%$ in 2013 vs 2008 . Overall, the percentages of patients with an $\mathrm{A} 1 \mathrm{C}<7 \%$ and $<8 \%$ were $>64 \%$ and $>85 \%$, respectively, in 2008 
and 2013. These percentages of patients with adequate glycemic control are significantly higher than what has been reported from the NHANES database; this observation may be, in part, because an integrated healthcare delivery model may assist in overcoming many of the barriers that have historically prevented obtaining glycemic control in patients with T2D. However, further studies comparing patients managed within an integrated delivery system versus a control group from a non-integrated model would be required to test this hypothesis. These findings are important, as identifying and overcoming the barriers to achieving glycemic targets (A1C) will become ever-important in the coming years, for preventing diabetes-related complications, as well as to ensure reimbursement for the medical care that is rendered to patients with diabetes as integrated health systems assume greater shared financial risk in newer payment models. A1C control is one of the quality measures used by the Center for Medicare and Medicaid Services for determining reimbursement rates to Accountable Care Organizations and incentives for the meaningful use of EHRs (http://www.cms.gov).

Hypercholesterolemia is a common comorbidity in patients with T2D, and an independent risk factor for CVD. Lipid control substantially reduces CVD, the major cause of death for individuals with diabetes. Most patients with diabetes meet the ADA recommendations for statin therapy: those with documented CVD, those aged $>40$ years with one or more additional cardiovascular risk factors (family history of CVD, HTN, smoking, dyslipidemia, albuminuria), or those lower-risk patients with an LDL $>100 \mathrm{mg} / \mathrm{dL}$, or those with multiple CVD risk factors. ${ }^{10}$ In this population, the mean LDL of both the 2008 and 2013 populations with T2D was $<100 \mathrm{mg} / \mathrm{dL}$, and $67.1 \%$ and $68.5 \%$, respectively, were receiving statin therapy. This is higher than the percentage of patients $(51.4 \%)$ reported to be receiving statin therapy, from 2007 to 2010, as per a recent analysis of NHANES data. ${ }^{4}$

HTN is another common comorbidity of patients with T2D. In this population, $82.5 \%$ and $87.2 \%$ of patients had documented HTN, among whom the mean systolic and diastolic BPs were below the ADA recommended goal BP $(<130 / 80),{ }^{12}$ recently changed to $<140 / 80 .^{10}$ Our estimation of the prevalence of HTN among these patients with diabetes is slightly higher than what was reported in the latest Centers for Disease Control and Prevention (CDC) Diabetes Fact Sheet (2014) from 2009 to 2012, where in adults aged 18 years or older with diagnosed diabetes, $71 \%$ were reported to have a BP greater than or equal to $140 / 90 \mathrm{~mm} \mathrm{Hg}$, or were receiving a prescription medication(s) to lower high BP. ${ }^{13}$

Perhaps the most interesting data derived from this report are the utilization of antidiabetic therapies among the patients with T2D. Not surprisingly, metformin and sulfonylureas remain the most commonly utilized antidiabetic therapies, likely because of their low cost. Overall, a decrease in the utilization of sulfonylureas, and an increase in the use of metformin were noted, however. These results are likely related to the recent recommendations by the ADA, the European Association for the Study of Diabetes, and the American Association of Clinical Endocrinologists to initiate metformin therapy as the first-line agent to manage glycemia, in the absence of contraindications or intolerability. In addition, reports have recently suggested that sulfonylureas impose increased cardiovascular risk vs metformin monotherapy. ${ }^{14}{ }^{15}$ The percentage of patients receiving metformin in combination with a sulfonylurea, however, was the same in 2008 and 2013. Not surprisingly, the use of thiazolidinediones has decreased considerably (overall, and in combination with metformin), which is clearly related to the safety concerns, risk, and contraindications/warnings that has surrounded these agents in recent years (cardiovascular risk with rosiglitazone and bladder cancer with pioglitazone) ${ }^{16-19}$ An increase in the use of DPP-4 inhibitors overall, and in combination with metformin, was also observed. It is possible that the changes in antidiabetic medications being prescribed in 2008 vs 2013 may, in part, explain the changes in glycemic control observed in this data set. Although GLP-1 agonist therapy has now been available for nearly 10 years in the USA, and more GLP-1 agonist therapies have become available since exenatide was originally approved in 2005, the percentage of patients receiving GLP-1 agonist therapy has not increased according to these data $(4.0 \%$ vs $3.8 \%$ in 2008 vs 2013).

The use of insulin therapy increased only slightly from $15.2 \%$ to $18.8 \%$, between 2008 and 2013 , respectively. The use of insulin premix formulations as well as human insulin decreased from 2008 to 2013. An increase in the use of analog and basal insulin was also observed.

The strengths of this study include the large number of participants identified, the use of the validated EMERGE algorithm ${ }^{9}$ to properly identify the participants, and the robust amount of clinical data which allowed for an extensive depiction of the participants. In addition, we had an A1C available in the vast majority of patients, only approximately $14 \%$ of whom did not have an available $\mathrm{A} 1 \mathrm{C}$ within 1 year prior to the index dates. However, this study is not without limitation. The biggest limitation is the inability to systematically extract data from the EHR that was not entered in a structured format (ie, clinical documentation in free-text progress notes). A significant amount of laboratory data from outside facilities was likely embedded within our clinician progress notes. As a result, not every patient had an A1C measure available in a structured format within 1 year prior to the index date. Patients without an A1C were excluded from any analysis that involved A1C. The same treatment was used for other covariates as well. In addition, with the exception of nephropathy, we relied heavily on the ICD-9 documentation of DM to ascertain microvascular complications. Many providers simply enter the code 250.02, T2D uncontrolled, uncomplicated, even if a patient has 
microvascular complications. Compared with estimates derived from NHANES, we are significantly underestimating the prevalence of microvascular complications. Most significant is the underestimation of the prevalence of retinopathy. We reported the prevalence of retinopathy to be around 3\%; however, from 2005 to 2008, the estimated prevalence of diabetic retinopathy in the USA, among US adults with diabetes aged 40 years and older, has previously been reported to be $28.5 \% .{ }^{20}$ Our estimate for the prevalence of neuropathy is also well below the estimates provided by the CDC National Diabetes Fact Sheets. $^{5}{ }^{13}$ These underestimations are expected to be related to inconsistent ICD-9 documentation of the microvascular complications, and with respect to retinopathy, because our ophthalmology department was not fully integrated into our EHR until 2012. Thus, until recently, the coordinated recording of dilated eye examinations within the EHR was not routinely performed. Our estimates of nephropathy (as per the DCSI definition) were within the expected range $(20-30 \%),{ }^{21}$ largely because this microvascular complication can be ascertained via laboratory data in addition to ICD-9 codes.

\section{CONCLUSIONS}

This analysis characterizing the population of patients with T2D within an integrated health delivery system underscores the complexity of treating these patients, as well as managing their glycemic control and comorbidities. While the prevalence of HTN has increased, the prevalence of other comorbidities decreased in 2013 when compared with 2008. The majority $(>64 \%)$ of patients had an $\mathrm{A} 1 \mathrm{C}<7 \%$, and $85 \%$ had an $\mathrm{A} 1 \mathrm{C}<8 \%$. In 2008 and 2013, metformin therapy was the most commonly utilized antidiabetic agent, and sulfonylureas were the most commonly utilized oral antidiabetic agent in combination with metformin. The ability to detect microvascular complications of diabetes from the systematic extraction of data from an EHR has significant limitations; the use of natural language processing technology may be helpful in capturing this information more reliably. An accurate capture of patient complexity (presence of comorbidities and complications) and their state of health in the EHR, coupled with the ability to use EHR data to predict outcomes, will become more important as the reimbursement model for medical care in the USA shifts away from a fee-for-service based system.

\section{Author affiliations}

${ }^{1}$ Department of Endocrinology, Cleveland Clinic Cleveland, Cleveland, Ohio, USA

${ }^{2}$ Novo Nordisk Inc, Plainsboro, New Jersey, USA

${ }^{3}$ Wake Forrest School of Medicine, Winston-Salem, North Carolina, USA

${ }^{4}$ Department of Health Economics and Outcomes Research, Novo Nordisk Inc, Plainsboro, New Jersey, USA

${ }^{5}$ Department of Quantitative Health Sciences, Cleveland Clinic, Cleveland, Ohio, USA

${ }^{6}$ Department of Medical Affairs, Novo Nordisk Inc, Plainsboro, New Jersey, USA
Acknowledgements The authors kindly thank Ed Kimball, Ph.D., of Novo Nordisk for his help in preparing and coordinating the authoring of the manuscript.

Contributors KMP researched the data and wrote the manuscript. TMH and SXK contributed to the discussion and reviewed/edited the manuscript. BJW researched and analyzed the data, designed the analysis, and contributed to the discussion. MWK, JB and JMB researched the data and reviewed/edited the manuscript. $\mathrm{CY}$ and BS researched and analyzed the data. AM extracted, researched, and analyzed the data. WW researched and analyzed the data and contributed to the discussion. RSZ researched the data, contributed to the discussion, and reviewed/edited the manuscript.

\section{Funding Novo Nordisk Research Grant.}

Competing interests KMP reports receiving research funding from Novo Nordisk and Merck; receiving consulting fees from Eli Lilly and Merck; and participating in the speaker bureaus of Eli Lilly, Merck, AstraZeneca, and Bristol Myers Squibb within the past 12 months. RSZ reports receiving research funding from Novo Nordisk and Merck; and participating in the speaker bureaus of Johnson \& Johnson and Merck within the past 12 months. BJW reports receiving research funding from Novo Nordisk and Merck within the past 12 months. TMH, SXK, JB, BS, and WW report being employees of Novo Nordisk.

Ethics approval This study was approved by the Cleveland Clinic's Institutional Review Board.

Provenance and peer review Not commissioned; externally peer reviewed.

Data sharing statement No additional data are available.

Open Access This is an Open Access article distributed in accordance with the Creative Commons Attribution Non Commercial (CC BY-NC 4.0) license, which permits others to distribute, remix, adapt, build upon this work noncommercially, and license their derivative works on different terms, provided the original work is properly cited and the use is non-commercial. See: http:// creativecommons.org/licenses/by-nc/4.0/

\section{REFERENCES}

1. National Center for Health Statistics, Division of Health Interview Statistics. In: National Center for Chronic Disease Prevention and Health Promotion, ed. Crude and age-adjusted percentage of civilian noninstitutionalized adults with diagnosed diabetes, United States, 1980-2010. Atlanta, GA: Centers for Disease Control and Prevention, Division of Diabetes Translation, 2012.

2. http://www.cdc.gov/nchs/nhanes/about_nhanes.Htm (accessed 12 Oct 2014).

3. Cheung BMY, Ong KL, Cherny SS, et al. Diabetes prevalence and therapeutic target achievement in the United States, 1999 to 2006. Am J Med 2009;122:443-53.

4. Stark Casagrande S, Fradkin JE, Saydah SH, et al. The prevalence of meeting $\mathrm{A} 1 \mathrm{C}$, blood pressure, and LDL goals among people with diabetes, 1988-2010. Diabetes Care 2013;36:2271-9.

5. Centers for Disease Control and Prevention. National diabetes fact sheet: National estimates and general information on diabetes and prediabetes in the United States, 2011. Atlanta, GA: US Department of Health and Human Services, Centers for Disease Control and Prevention, 2011.

6. Ali MK, Bullard KM, Saaddine JB, et al. Achievement of goals in US diabetes care, 1999-2010. N Engl J Med 2013;368:1613-24.

7. Gregg EW, Li Y, Wang J, et al. Changes in diabetes-related complications in the United States, 1990-2010. N Engl J Med 2014;370:1514-23.

8. Enthoven AC. Integrated delivery systems: the cure for fragmentation. Am J Manag Care 2009;15(10 Suppl):S284-90.

9. Kho AN, Hayes MG, Rasmussen-Torvik L, et al. Use of diverse electronic medical record systems to identify genetic risk for type 2 diabetes within a genome-wide association study. J Am Med Inform Assoc 2012;19:212-18.

10. American Diabetes Association. Standards of medical care in diabetes-2014. Diabetes Care 2014; 37(Suppl 1):S14-80.

11. Young BA, Lin E, Von Korff M, et al. Diabetes complications severity index and risk of mortality, hospitalization, and healthcare utilization. Am J Manag Care 2008;14:15-23. 
12. American Diabetes Association. Standards of medical care in diabetes-2011. Diabetes Care 2011;34(Suppl 1):S11-61.

13. Centers for Disease Control and Prevention. National Diabetes Statistics Report: estimates of diabetes and its burden in the United States, 2014. Atlanta, GA: US Department of Health and Human Services, 2014.

14. Pantalone KM, Kattan MW, Yu C, et al. Increase in overall mortality risk in patients with type 2 diabetes receiving glipizide, glyburide or glimepiride monotherapy versus metformin: a retrospective analysis. Diabetes Obes Metab 2012;14:803-9.

15. Johnson JA, Simpson SH, Toth EL, et al. Reduced cardiovascular morbidity and mortality associated with metformin use in subjects with type 2 diabetes. Diabet Med 2005;22:497-502.

16. Nissen SE, Wolski K. Effect of rosiglitazone on the risk of myocardial infarction and death from cardiovascular causes. N Engl J Med 2007;356:2457-71.
17. Azoulay L, Yin $\mathrm{H}$, Filion KB, et al. The use of pioglitazone and the risk of bladder cancer in people with type 2 diabetes: nested case-control study. BMJ 2012;344:e3645.

18. Dormandy JA, Charbonnel B, Eckland DJ, et al. PROactive investigators. Secondary prevention of macrovascular events in patients with type 2 diabetes in the PROactive study (PROspective pioglitAzone clinical trial in macroVascular events): a randomised controlled trial. Lancet 2005;366:1279-89.

19. Lewis JD, Ferrara A, Peng T, et al. Risk of bladder cancer among diabetic patients treated with pioglitazone: interim report of a longitudinal cohort study. Diabetes Care 2011;34:916-22.

20. Zhang X, Saaddine JB, Chou CF, et al. Prevalence of diabetic retinopathy in the United States, 2005-2008. JAMA 2010;304:649-56.

21. Molitch ME, DeFronzo RA, Franz MJ. Nephropathy in diabetes. Diabetes Care 2004;27(Suppl 1):s79-83. 